\title{
Effects of anabolic steroids on diaphragm impairment induced by methylprednisolone in emphysematous hamsters
}

\author{
R.H.H. van Balkom*, P.N.R. Dekhuijzen*, H.F.M. van der Heijden*, H.T.M. Folgering*, \\ J.A.M. Fransen**, C.L.A. van Herwaarden*
}

Effects of anabolic steroids on diaphragm impairment induced by methylprednisolone in emphysematous hamsters. R.H.H. van Balkom, P.N.R. Dekhuijzen, H.F.M. van der Heijden, H.T.M. Folgering, J.A.M. Fransen, C.L.A van Herwaarden. (C)ERS Journals Ltd 1999. ABSTRACT: This study was designed to investigate whether the administration of the anabolic steroid nandrolone decanoate is able to antagonize the loss in diaphragm function induced by long-term administration of a low-dose of methylprednisolone in emphysematous hamsters.

Normal and emphysematous male hamsters were randomized to receive either saline or methylprednisolone $0.2 \mathrm{mg} \cdot \mathrm{kg}^{-1} \cdot \mathrm{day}^{-1}$ for 9 months, with or without nandrolone decanoate $1 \mathrm{mg} \cdot \mathrm{kg}^{-1} \cdot \mathrm{week}^{-1} \mathrm{i}$. m. during the final 3 months. Diaphragm contractile properties and myosin heavy chain composition were determined.

Compared to control hamsters, the force generating capacity of isolated diaphragm strips decreased by approximately $12 \%$ in the emphysema group and by approximately $22 \%$ in the emphysema plus methylprednisolone group. Addition of nandrolone decanoate to the emphysema plus methylprednisolone hamsters significantly improved force generation. The atrophy of type IIa and IIx diaphragm fibres in the emphysema plus methylprednisolone group was completely reversed to the level of control hamsters by the addition of nandrolone decanoate.

In conclusion, nandrolone decanoate in part reversed the loss in diaphragm forcegenerating capacity in emphysematous hamsters treated with methylprednisolone, and reversed type IIa and IIx fibre atrophy completely.

Eur Respir J 1999; 13: 1062-1069.
*Dept of Pulmonary Diseases, University Hospital Nijmegen, the Netherlands. **Dept of Cell Biology and Histology, University of Nijmegen, the Netherlands.

Correspondence: P.N.R. Dekhuijzen

Dept of Pulmonary Diseases

University Hospital Nijmegen

PO Box 9101

6500 HB Nijmegen

The Netherlands

Fax: 313243540788

Keywords: Animals contractile properties corticosteroids

immunohistochemistry nandrolone decanoate respiratory muscles

Received: March 101998

Accepted after revision December 301998

This study was supported with a grant from the Dutch Asthma Foundation (No. 92.17).
Patients with severe chronic obstructive pulmonary disease (COPD) may suffer from respiratory muscle weakness due to hyperinflation, malnutrition, disturbances in blood gases and cardiac failure [1]. In these patients, respiratory muscle function was recently reported to be further reduced by administration of daily low-dose methylprednisolone (MP) (mean dosage $4.3 \mathrm{mg} \cdot \mathrm{day}^{-1}$ ) for 6 months [2]. Since respiratory muscle weakness may contribute to respiratory failure, interventions that attenuate or even abolish respiratory muscle impairment in these patients may be of clinical importance.

With respect to the impairment caused by glucocorticoids, the use of anabolic steroids may be considered as attenuating or preventive agents. Anabolic steroids are able to raise skeletal muscle protein synthesis [3]. This may be important since the major cause of glucocorticoid-induced muscle dysfunction is believed to be a reduction in muscle protein [4]. Besides glucocorticoid treatment, malnutrition is also known to result in a catabolic condition in patients with COPD $[5,6]$. This catabolic condition may in part be reversed by anabolic steroids in combination with adequate protein intake [3]. Indeed, ScHols et al. [7] recently showed that anabolic steroids combined with refeeding improved respiratory muscle function in undernourished COPD patients. Whether anabolic steroids are also able to counteract the glucocorticoid-induced impair- ment in human respiratory muscle function has not yet been studied.

Animal models have been used to obtain an insight into the underlying mechanisms responsible for the changes in diaphragm muscle structure and function. The type and severity of alterations caused by glucocorticoids appear to depend on the type of steroid used, and the dosage and the duration of administration. In a prior study, changes were observed in contractile properties and morphology of the diaphragm from normal rats following long-term treatment with a low dose of MP $\left(0.2 \mathrm{mg} \cdot \mathrm{kg}^{-\mathrm{P}} \cdot \mathrm{day}^{-1}\right.$ for 6 months $)$ [8]. Recent results have shown that in normal rats, anabolic steroids are able to reverse diaphragm function loss from corticosteroid-induced myopathy [9].

The use of emphysematous animals to determine drug effects on the diaphragm may be of interest since this animal model resembles to some extent the functional alterations observed in COPD patients. Induction of emphysema by intratracheal instillation of elastase in hamsters results in a progressive increase in lung volumes and compliance [10], diaphragm muscle adaptation to hyperinflation of the lungs $[11,12]$, and impairment in respiratory muscle function $[11,12]$.

Therefore, this emphysematous model was used to investigate whether anabolic steroids in a clinically relevant dose are able to antagonize the impairment caused 
by long-term low-dose MP treatment on the diaphragm which has adapted to emphysematous changes in the lung. Based on the metabolic effects of anabolic steroids described above, it was hypothesized that anabolic steroids will be able to reverse the alterations in diaphragm function and structure induced by MP. Therefore, the contractile properties of the diaphragm of emphysematous hamsters treated with nandrolone decanoate (ND) during the final 3 months of a 9-month treatment period with a low dose of MP were examined. Immunoreactivity to myosin heavy chain (MHC) antibodies was tested to evaluate the effects of MP and ND on diaphragm fibre composition.

\section{Methods}

\section{Study design, induction of emphysema, and treatment}

Adult male Golden hamsters $(n=48)$, aged $>40$ weeks, weighing $\sim 150 \mathrm{~g}$, were lightly anaesthetized with a mixture of halothane and nitrous oxide, vaporized in air. A polyethylene cannula was inserted into the trachea with the tip located above the carina. The hamsters received either a single instillation of $\mathrm{NaCl} 0.9 \%$ at $0.5 \mathrm{~mL} \cdot 100 \mathrm{~g}$ body weight $^{-1}(\mathrm{n}=12)$ or $25 \mathrm{IU} \cdot 100 \mathrm{~g}$ body weight ${ }^{-1}$ porcine pancreatic elastase (PPE) (Sigma Chemicals, Bornem, Belgium $)(n=36)$. PPE was dissolved at a concentration of 50 $\mathrm{IU} \cdot \mathrm{mL}^{-1}$ in $0.9 \% \mathrm{NaCl}$ to ensure similar fluid loads per animal. To improve the distribution to the peripheral parts of the lung, $3 \mathrm{~mL}$ of room air was injected through the tube. The hamsters were monitored carefully until spontaneous breathing.

Normal hamsters were divided into two groups 6 months after instillation with $\mathrm{NaCl}$ (fig. 1): controls receiving saline $0.2 \mathrm{~mL}$ s.c. daily $(\mathrm{n}=12)$; and control hamsters receiving MP hemisuccinate (Sigma Chemicals) $0.2 \mathrm{mg} \cdot \mathrm{kg}^{-1}$ s.c. daily for 9 months $(\mathrm{n}=12)$.

Six months after PPE instillation, emphysematous hamsters were randomized into two groups (fig. 1): emphysematous hamsters (Emph) receiving saline $0.2 \mathrm{~mL}$ s.c. daily $(\mathrm{n}=12)$; and emphysematous hamsters receiving MP $0.2 \mathrm{mg} \cdot \mathrm{kg}^{-1}$ s.c. daily $(\mathrm{n}=24)$. After 6 months of MP treatment, these animals were subdivided into two further groups: those continuing with MP $0.2 \mathrm{mg} \cdot \mathrm{kg}^{-1}$ s.c. daily until the end of the study (Emph+MP) $(n=12)$; and those continuing with MP $0.2 \mathrm{mg} \cdot \mathrm{kg}^{-1}$ s.c daily until the end of

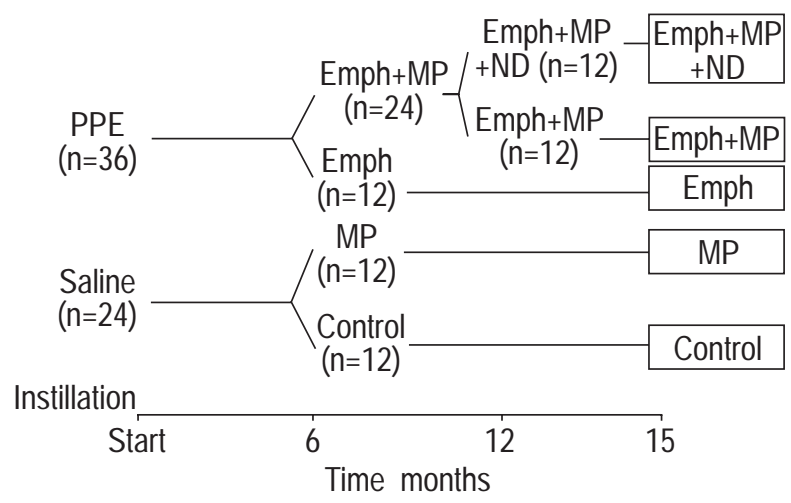

Fig. 1. - Time course of intervention and experimental design. PPE: porcine pancreatic elastase. Emph: elastase-induced emphysema; MP: methylprednisolone; ND: nandralone decanoate. the study, but in combination with ND (Organon, Oss, the Netherlands) for the final 3 months of the study at 1 $\mathrm{mg} \cdot \mathrm{kg}^{-1}$ i.m. once a week (Emph+MP+ND).

The intention was to evaluate the effects of a low dose of a nonfluorinated steroid (i.e. MP) comparable to the dose occasionally used in the chronic treatment of patients with COPD. A calculated estimation showed that $0.2 \mathrm{mg} \cdot \mathrm{kg}^{-1}$ MP is equivalent to a dose of at most $14 \mathrm{mg} \cdot$ day $^{-1}$ in a 70 $\mathrm{kg}$ human. This was based on similar anti-inflammatory potency and metabolism in rodents and humans [13, 14], an absorption of only $60 \%$ after $i . m$. injection of cortisone acetate [15], and the observation that the s.c. route requires higher doses to produce similar effects compared to i.m. administration [16]. Indeed, in a previous study, administration of $0.2 \mathrm{mg} \cdot \mathrm{kg}^{-1} \cdot \mathrm{day}^{-1} \mathrm{MP}$ for 6 months in normal rats caused a $10-15 \%$ reduction in diaphragm twitch and maximum tetanic force generation [8]. Although the therapeutic efficacy of glucocorticoids in COPD is controversial [5], prolonged prednisolone administration in doses of $10-15 \mathrm{mg}$ daily are no exception in the treatment of patients. ND was administered in a dose used by others in clinical studies [7], and as recommended for use in patients by the manufacturer.

During the 9 month treatment period, all hamsters received a similar volume $(\sim 0.20 \mathrm{~mL})$ with each s.c. injection. The hamsters were fed ad libitum (RMHB, Hope Farms, Woerden, The Netherlands) with free access to drinking water, under a 12/12 hour light-dark regimen and weighed once every week. Although daily food intake was not precisely quantified (animals were not held in metabolic cages), food intake appeared to be similar in all groups $\left(\sim 10 \mathrm{~g} \cdot \mathrm{day}^{-1}\right)$. In line with earlier studies $[17,18]$, previous pilot experiments showed no change in the body weight curve in normal and Emph hamsters. Therefore, a pair-weight or pair-fed control group was not included.

Fifteen months after PPE instillation and 9 months following the start of the treatment with MP or saline, the animals were sacrificed to measure contractile properties and histological characteristics of the diaphragm. The diaphragm, musculus extensor digitorum longus (EDL) and the soleus muscle were weighed immediately after dissection and the lungs were removed to evaluate the extent of emphysema. The study was approved by the Animal Experiments Committee of the University of Nijmegen and performed according to the Dutch National Guidelines of Animal Care.

\section{Verification of emphysema}

Five animals per group were used to evaluate the degree of emphysema induced by PPE, as the degree of emphysema had been found to be well reproducible in a pilot study. At the end of the treatment period, the hamsters were anaesthetized with sodium pentobarbital $\left(70 \mathrm{mg} \cdot \mathrm{kg}^{-1}\right.$ i.p.). A polyethylene cannula was inserted through a tracheotomy for mechanical ventilation (oxygen-enriched gas mixture, flow $0.5 \mathrm{~mL} \cdot \mathrm{g}$ body weight ${ }^{-1} \cdot \mathrm{min}^{-1}$, respiratory frequency 70 breaths $\cdot \mathrm{min}^{-1}$ and a duty cycle of $50 \%$ ). After dissection, the lungs were inflated with $4 \%$ buffered formalin to a pressure of $25 \mathrm{cmH}_{2} \mathrm{O}$. The minimal fixation time was 2 h. Postfixation lung volume was determined by fluid displacement. The left lower lobe was embedded in 
paraffin and sagittal sections ( $6 \mu \mathrm{m}$ thickness) were cut and stained with haematoxylin-eosin. Alveolar cross-sectional area (CSA) was measured to determine the extent of emphysematous changes in the lung. The measurements were performed on the left lower lobe since a pilot study had shown a good correlation between the CSA of the alveoli of this lobe and the lung volume measured by fluid displacement. These measurements were made using a Sprynt-based, PC-Image digital analysis system (Bos Inc, Waddinxveen, the Netherlands).

\section{Contractile properties}

Procedures for anaesthesia and intubation were performed as described above. A combined laparotomy and thoracotomy was performed to remove the diaphragm. Immediately after excision, the diaphragm was immersed in a cooled, oxygenated Krebs' solution at a $\mathrm{pH}$ of 7.4. This solution consisted of $\left(\mathrm{mmol} \cdot \mathrm{L}^{-1}\right), 137 \mathrm{NaCl}, 4 \mathrm{KCl}, 1$ $\mathrm{MgCl}_{2}, 1 \mathrm{KH}_{2} \mathrm{PO}_{4}, 24 \mathrm{NaHCO}_{3}, 2 \mathrm{CaCl}_{2}$, and 7 glucose. D-tubocurarine chloride $25 \mu \mathrm{M}$ (Sigma Chemicals) was added to prevent spontaneous neuromuscular activity. Contractile properties were measured on two small rectangular bundles, dissected from the middle part of the lateral costal region of each hemidiaphragm and parallel to the long axis of the muscle fibres. Silk sutures were firmly tied to both ends of the bundle to serve as anchoring points. Each bundle was placed in a tissue bath containing Krebs at $37^{\circ} \mathrm{C}$ and was oxygenated with $95 \% \mathrm{O}_{2}$ and $5 \% \mathrm{CO}_{2}$. The central tendon insertion of the bundles were tied to a fixed point and the costal margin origin to an isometric force transducer (Sensotec, model 31/1437, Columbus, OH, USA). Data acquisition and storage were performed using a Dash-16 interface and Twist-Trigger software (IDelectronics, University of Nijmegen, the Netherlands). The stimulator (ID-electronics) was activated by a personal computer. The muscle strips were stimulated with two large platinum electrodes on both sides of the muscle. To ensure supramaximal stimulation, subsequent stimulations were performed $20 \%$ above the voltage at which maximal forces were obtained. The pulse duration was set to $0.2 \mathrm{~ms}$. Twitch stimuli were used to determine the optimal length $(L o)$, followed by a 15 min thermo-equilibration period. The following measurements were made.

Twitch characteristics. Two twitches were recorded at $L o$ to obtain maximal twitch force $(P \mathrm{t})$, contraction time $(\mathrm{C} t)$ and half relaxation time $(1 / 2 \mathrm{R} t)$. The averages were used for further analysis.

Maximal tetanic contraction. Two maximal tetanic stimuli (with a frequency of $160 \mathrm{~Hz}$ and a train duration of $400 \mathrm{~ms}$ ) were generated to obtain maximal tetanic force $(P \max )$.

Force-frequency protocol. Muscle bundles were stimulated every $2 \mathrm{~min}$ with the following frequencies: 25 , $50,80,120$ and $160 \mathrm{~Hz}$ (train duration $400 \mathrm{~ms}$ ). The generated force was expressed per CSA $\left(\mathrm{N} \cdot \mathrm{cm}^{-2}\right)$. CSA was measured by dividing diaphragm bundle weight by muscle density $\left(1.056 \mathrm{mg} \cdot \mathrm{mm}^{-3}\right)$ and bundle length. Diaphragm bundle dimensions were measured using an analogue graduated ruler (Mitutoyo model 560-182 Veenendaal, the Netherlands)

\section{Morphometric evaluation of the diaphragm muscle}

Resting (excised) muscle length of strips obtained from the costal part of the right hemidiaphragm were measured. Before freezing, the strips were stretched to 1.5-times this excised length to approximate $L_{0}$ [19], and pinned on a cork backing. The specimens were quickly frozen in isopentane cooled in liquid nitrogen followed by further freezing in liquid nitrogen. Serial cross sections were cut at $7 \mathrm{~mm}$ with a cryostat kept at $-30^{\circ} \mathrm{C}$.

Myosin heavy chain antibodies (DSM, Braunschweig, Germany) were used for morphometric examination of serial diaphragm sections. The following antibodies were used: BA-D5 reactive with MHC-I, SC-71 reactive with MHC-IIa, BF-35 reactive with MHC-I, MHC-IIa and MHC-IIb but not with MHC-IIx, and BF-F3 reactive with MHC-IIb [20]. Incubation with anti-myosin heavy chain antibodies was performed at room temperature for $1 \mathrm{~h}$. Antibodies were subsequently labelled with ultra-small immunogold reagent followed by silver enhancement (Aurion, Wageningen, the Netherlands). The CSA of at least 250 fibres were analysed from each diaphragm using the Sprynt-based, PC-Image digital analysis system (Bos Inc).

\section{Data analysis}

Data of contractile properties of the two bundles obtained from one hamster were averaged. The SPSS/ PC + package version 6.1.3 (Chicago, IL, USA) was used for statistical analysis. Data were compared using one-way analysis of variance (ANOVA) followed by Duncan's multiple-range test. Repeated measures ANOVA was used to analyse the force-frequency relationship. Morphometric analysis was performed using the average per fibre type per animal which was utilized as a single value in the statistical analysis. Results were considered significant at $p<0.05$. All data were expressed as mean \pm SEM.

\section{Results}

\section{Body and muscle weights}

The growth curve for the hamsters was not significantly affected by drug treatment or by induction of emphysema. Initial and final body weights were similar in all groups (table 1). No differences in diaphragm and soleus muscle weights were observed. An increase in EDL muscle weight, normalized for body weight, was found in the Emph+MP+ND group compared to the control, Emph, and Emph+MP groups (table 1).

\section{Degree of emphysema}

The alveolar CSA in the Emph, Emph+MP and Emph+MP+ND hamsters was $\sim 190 \%$ that of control and MP hamsters $\left(5,061 \pm 319,5,132 \pm 328\right.$ and 5,248 $\pm 363 \mu^{2}$, respectively, versus $2,740 \pm 86 \mu^{2}$ and $2,675 \pm 79 \mu \mathrm{m}^{2}$ in control and MP). Fluid displacement lung volume increased by $\sim 50 \%$ from normal hamsters $(9.5 \pm 0.8,9.7 \pm 1.2$ and $9.8 \pm 1.3 \mathrm{~mL}$ in Emph, Emph+MP and Emph+MP+ND 
Table 1. - Body and muscle weights

\begin{tabular}{lcccc}
\hline Treatment & $\begin{array}{c}\text { Initial body weight } \\
\text { g }\end{array}$ & $\begin{array}{c}\text { Final body weight } \\
\text { g }\end{array}$ & $\begin{array}{c}\text { Diaphragm } \\
\text { \%o body weight }\end{array}$ & $\begin{array}{c}\text { EDL } \\
\text { \%o body weight }\end{array}$ \\
\hline Control & $150 \pm 3$ & $149 \pm 2$ & $2.02 \pm 0.04$ & $0.21 \pm 0.01$ \\
MP & $151 \pm 4$ & $145 \pm 3$ & $1.94 \pm 0.03$ & $0.22 \pm 0.01$ \\
Emph & $149 \pm 3$ & $148 \pm 3$ & $2.08 \pm 0.02$ & $0.20 \pm 0.01$ \\
Emph+MP & $152 \pm 4$ & $147 \pm 3$ & $2.04 \pm 0.03$ & $0.24 \pm 0.01$ \\
Emph+MP+ND & $149 \pm 4$ & $145 \pm 3$ & $2.07 \pm 0.04$ & $0.22 \pm 0.01$ \\
\hline
\end{tabular}

Data are presented as mean \pm SEM. EDL: extensor digitorum longus. Experimental groups are: Control, receiving saline for 9 months; MP, receiving methylprednisolone (MP) for 9 months; Emph, elastase-induced emphysematous hamsters receiving saline for 9 months; Emph+MP, elastase-induced emphysematous hamsters receiving MP for 9 months; Emph+MP+ND, elastase-induced emphysematous hamsters receiving 9 months of MP treatment with nandrolone decanoate (ND) added for the final 3 months. *: p<0.05 compared to all other groups.

versus $6.3 \pm 0.5 \mathrm{~mL}$ and $6.5 \pm 0.5 \mathrm{~mL}$ in control and $\mathrm{MP})$. No differences were found between the three groups of emphysematous hamsters.

\section{Diaphragm bundle dimensions and contractile proper- ties}

Diaphragm muscle strip length was significantly reduced in all Emph groups compared to the control and MP groups (table 2). Muscle strip weight was reduced only in the Emph group. However, no differences were found between the groups when muscle strip weight was normalized for muscle strip dimension (table 2).

$P \mathrm{t}$ was reduced in the diaphragm of the MP, Emph and Emph+MP hamsters compared to control and Emph+ $\mathrm{MP}+\mathrm{ND}$ (fig. 2a). Twitch force in the MP hamsters was significantly lower than in Emph. Addition of ND increased $P \mathrm{t}$ to the level of control hamsters. $P_{\mathrm{o}}$ was significantly lower in all intervention groups (fig. $2 b$ ). No significant differences in contractile properties were found between MP and Emph+MP. Since $P$ max decreased in the MP and Emph+MP group compared to all other groups, the most pronounced reduction in maximum force was observed following MP treatment. Administration of ND to the animals increased $P \max$ to the level of the Emph group (fig. 2b). Neither induction of emphysema nor treatment with MP or ND caused changes in $\mathrm{C} t$ or $1 / 2 \mathrm{R} t$.

Repeated measures ANOVA indicated a significant effect of treatment on the absolute and normalized force-frequency relationship (fig. 3). Force generation at $25 \mathrm{~Hz}$ was decreased in the MP and Emph+MP group compared to control hamsters but not compared to Emph hamsters. At all stimulation frequencies $>25 \mathrm{~Hz}$, no differences in force between the MP and Emph+MP groups were found. However, force production in the Emph+MP group was significantly lower than in Emph hamsters. Addition of ND reversed this decrease in force to the level of the
Emph group. No differences were found between the control, MP, Emph and Emph+MP groups when forces were normalized for maximum tetanic tension. However, ND addition seemed to have a more pronounced effect at lower frequencies as reflected by the significant increase in $P \mathrm{t} / P \max$ in the Emph+MP+ND compared to control, MP and Emph hamsters (table 3). Normalized force production at $25 \mathrm{~Hz}$ was significantly increased in the $\mathrm{Emph}+\mathrm{MP}+\mathrm{ND}$ group compared to the control group.

\section{Diaphragm muscle morphology}

The number of fibres expressing predominantly type I MHC isoforms decreased in the diaphragm of the Emph hamsters compared to that of the control and Emph+ $\mathrm{MP}+\mathrm{ND}$ hamsters (table 4). No significant changes in MHC-IIa and MHC-IIx fibre distribution were observed.

Both MHC-IIa and MHC-IIx fibre CSA were reduced in MP, Emph and Emph+MP diaphragm muscle compared to control (fig. 4). However, no significant difference was found between CSA in the Emph and the Emph+MP groups. In the Emph+MP+ND diaphragm, MHC-IIa and MHC-IIx CSA were significantly increased compared to the MP, Emph and Emph+MP groups, while no difference in fibre CSA was found compared to control hamsters.

Relative fibre type contribution to total diaphragm muscle area was not changed by either treatment (table 4). No fibres reactive with type IIb MHC were found.

\section{Discussion}

\section{Summary of the results}

The present study was designed to evaluate diaphragm muscle impairment caused by 9 months of low-dose MP administration in emphysematous hamsters. In addition,

Table 2. - Diaphragm muscle strip dimensions

\begin{tabular}{lccccc}
\hline Treatment & $\begin{array}{c}\text { Length } \\
\mathrm{mm}\end{array}$ & $\begin{array}{c}\text { Width } \\
\mathrm{mm}\end{array}$ & $\begin{array}{c}\text { Thickness } \\
\mathrm{mm}\end{array}$ & $\begin{array}{c}\text { Weight } \\
\mathrm{mg}\end{array}$ & $\begin{array}{c}\text { Weight normalized for } \\
\text { strip dimension } \mathrm{mg} \cdot \mathrm{mm}{ }^{-3}\end{array}$ \\
\hline Control & $15.6 \pm 0.3$ & $2.1 \pm 0.03$ & $0.6 \pm 0.01$ & $15.3 \pm 0.7$ & $0.8 \pm 0.02$ \\
MP & $15.2 \pm 0.3$ & $2.2 \pm 0.05$ & $0.6 \pm 0.02$ & $15.9 \pm 0.8$ & $0.8 \pm 0.03$ \\
Emph & $14.2 \pm 0.2^{*}$ & $2.0 \pm 0.02$ & $0.6 \pm 0.01$ & $12.9 \pm 0.5^{*}$ & $0.8 \pm 0.03$ \\
Emph+MP & $14.5 \pm 0.1^{*}$ & $2.1 \pm 0.03$ & $0.6 \pm 0.01$ & $14.1 \pm 0.6$ & $0.8 \pm 0.02$ \\
Emph+MP+ND & $14.6 \pm 0.3^{*}$ & $2.0 \pm 0.03$ & $0.6 \pm 0.01$ & $14.1 \pm 0.5$ & $0.8 \pm 0.02$ \\
\hline
\end{tabular}

Data are presented as mean \pm SEM. For definitions of experimental groups see footnote to table $1 .^{*}: \mathrm{p}<0.05$ compared to control and MP. 

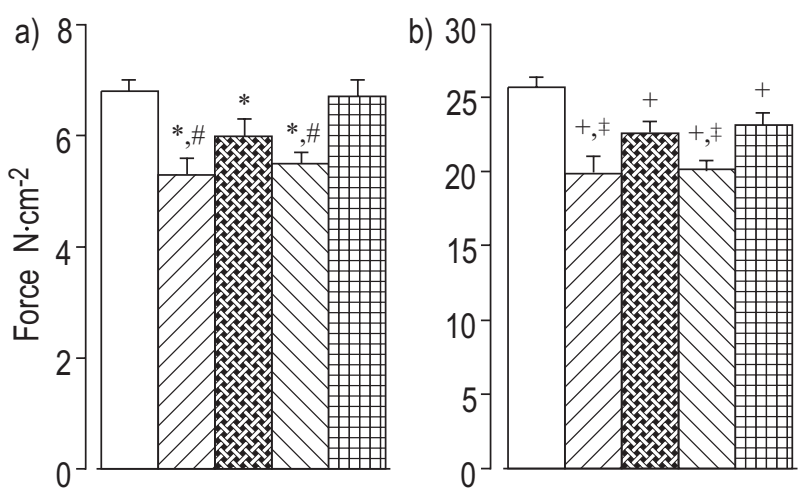

Fig. 2. - a) Twitch force $(P \mathrm{f})$; and b) maximal tetanic force $(P \max )$ of the diaphragm. $\square$ : control; $\mathbb{Z}:$ MP; $:$ : Emph; $\mathbb{\nabla}$ : Emph+MP; 曲: $\mathrm{Emph}+\mathrm{MP}+\mathrm{ND}$. For explanation of treatment groups see figure 1. Data are shown as mean \pm SEM. $*$ : $p<0.05$ compared to control and Emph+MP+ $\mathrm{ND} ;{ }^{\#}: \mathrm{p}<0.05$ compared to Emph; ${ }^{+}: \mathrm{p}<0.05$ compared to control; ${ }^{\star}$ : $\mathrm{p}<0.05$ compared to Emph and Emph+MP+ND.

the ability of the anabolic steroid ND to antagonize the loss in diaphragm function caused by the combination of emphysema and long-term treatment with a low dose of MP was investigated.

The results show that, despite the decrease in force generation in the diaphragm of emphysematous hamsters, MP reduced force generation in the diaphragm of emphysematous and normal hamsters to a similar extent. Secondly, the data show that diaphragm function in the MP treated emphysematous hamsters improved following ND addition. At low stimulation frequencies, this improvement in force generation was up to the level of control hamsters, while maximum force generation improved to the level of force production in the Emph hamsters. The MHC-IIa and MHC-IIx fibre atrophy in the Emph+MP diaphragm was completely reversed to the level of controls by the addition of ND.

Effects of emphysema and methyl prednisolone on diaphragm function and structure

Hamsters have been frequently used to evaluate diaphragmatic adaptations to elastase-induced emphysema. The increase in alveolar CSA observed following induction of emphysema combined with an increase in fluid displacement by $\sim 50 \%$ in all three PPE-instilled groups indicated the presence of emphysematous changes in this study. Induction of emphysema may cause changes in diaphragm force generation $[11,12,21]$. The reduction in $P \mathrm{t}$ and $P \max$ in the present study was in line with previous reports in the hamster model $[11,12,21]$. This reduction in specific force can be the result of an increase in diaphragm muscle load, as a result of emphysematous changes in the lung [22]. Force-generating capacity was also reduced in models representing skeletal [23] and diaphragm muscle [24] overloading. In these overloaded muscles and also in the diaphragm of emphysematous hamster [11], the reduction in force was associated with fast fibre hypertrophy. However, in line with the present findings, muscle overload [25] and the induction of emphysema [26] can also decrease fibre CSA. Although emphysema did not change relative fibre type contribution to total diaphragm muscle area in the present or in
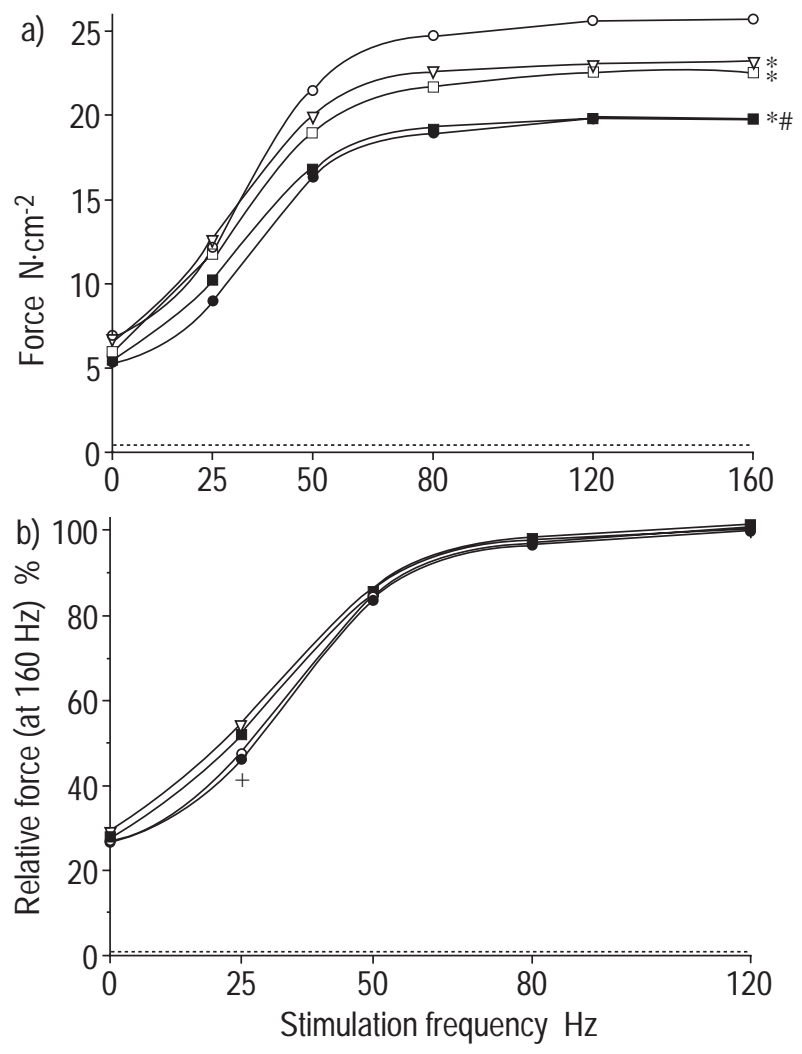

Fig. 3. - Force-frequency curves for a) absolute force; and b) normalized for maximal tetanic force. $\bigcirc$ : control; $\bullet$ : MP; $\square$ : Emph; $\mathbf{\square}$ Emph+MP; $\nabla:$ Emph+MP+ND. For explanation of treatment groups see figure 1. *: p $<0.05$ compared to control; ${ }^{*}: \mathrm{p}<0.05$ compared to Emph and Emph+ MP+ND; ${ }^{+}: \mathrm{p}<0.05$ for Emph+MP+ND compared to control. Dashed lines represent pooled standard error.

previous studies [11], MHC-IIx fibre CSA decreased by $\sim 20 \%$ in the diaphragm of the Emph hamsters. The observation that in the emphysematous diaphragm a decrease in force-generating capacity can be associated with fast muscle fibre hypertrophy and atrophy, indicates muscle changes at the ultrastructural level.

In the emphysematous diaphragm, FARKAS and Roussos [26] observed increased oxidative and decreased glycolytic activity, as shown by citrate synthase and phosphofructokinase, respectively. These biochemical changes following emphysema were associated with fast fibre atrophy. Besides these disadvantageous changes, more subtle alterations such as changes in muscle contractile protein composition, are also likely to be responsible for the decrease in force in the overloaded muscles.

Table 3. - Diaphragm contractile properties

\begin{tabular}{lccc}
\hline Treatment & $\begin{array}{c}\mathrm{C} t \\
\mathrm{~ms}\end{array}$ & $\begin{array}{c}1 / 2 \mathrm{R} t \\
\mathrm{~ms}\end{array}$ & $\begin{array}{c}P \mathrm{t} / P \max \\
\%\end{array}$ \\
\hline Control & $26.7 \pm 0.2$ & $28.4 \pm 0.7$ & $26.5 \pm 0.6$ \\
MP & $26.1 \pm 0.3$ & $28.1 \pm 0.7$ & $27.2 \pm 0.9$ \\
Emph & $26.7 \pm 0.4$ & $28.8 \pm 0.8$ & $26.8 \pm 0.6$ \\
Emph+MP & $27.0 \pm 0.3$ & $29.5 \pm 0.6$ & $27.9 \pm 0.8$ \\
Emph+MP+ND & $27.5 \pm 0.4$ & $30.8 \pm 1.0$ & $29.3 \pm 0.9^{*}$ \\
\hline
\end{tabular}

Data are presented as mean \pm SEM. C $t$ : contraction time; $1 / 2 \mathrm{R} t$ : half relaxation time; $P \mathrm{t}$ : maximal twitch force; $P$ max: maximal tetanic force. For definitions of experimental groups see footnote to table 1. *: p<0.05 compared to control and Emph. 
Table 4. - Fibre type distribution and relative fibre type contribution to the total diaphragm muscle area

\begin{tabular}{lccc}
\hline Treatment & $\begin{array}{c}\text { MHC-I } \\
\%\end{array}$ & $\begin{array}{c}\text { MHC-IIa } \\
\%\end{array}$ & $\begin{array}{c}\text { MHC-IIx } \\
\%\end{array}$ \\
\hline Fibre type distribution & & & \\
$\quad$ Control & $26.5 \pm 0.9$ & $41.4 \pm 2.4$ & $32.0 \pm 1.9$ \\
MP & $23.8 \pm 0.9$ & $31.5 \pm 2.5$ \\
Emph & $22.7 \pm 1.2^{*}$ & $44.8 \pm 1.9$ & $34.5 \pm 2.1$ \\
Emph+MP & $24.3 \pm 0.8$ & $42.8 \pm 1.9$ & $33.4 \pm 0.7$ \\
Emph+MP+ND & $26.4 \pm 0.8$ & $42.3 \pm 0.7$ & $32.2 \pm 1.1$ \\
Fibre type contribution to total diaphragm area & & $41.4 \pm 1.2$ & $50.4 \pm 2.5$ \\
Control & $17.4 \pm 0.7$ & $35.9 \pm 3.2$ \\
MP & $18.6 \pm 0.9$ & $32.2 \pm 2.3$ & $49.0 \pm 2.6$ \\
Emph & $17.5 \pm 1.0$ & $35.5 \pm 2.4$ & $49.2 \pm 1.0$ \\
Emph+MP & $18.1 \pm 0.7$ & $33.5 \pm 2.4$ & $50.3 \pm 1.3$ \\
Emph+MP+ND & $18.2 \pm 0.5$ & $32.7 \pm 0.7$ & $31.5 \pm 1.4$ \\
\hline
\end{tabular}

Data are presented as mean \pm SEM. For definitions of experimental groups see footnote to table 1 . MHC: myosin heavy chain. *: $p<0.05$ compared to control and Emph+MP+ND.

Following MP administration, twitch and tetanic forces further decreased in the diaphragm of the Emph hamsters. Morphometrically, type IIa fibre atrophy was found in the Emph+MP group compared to controls, but type IIx fibre size decreased to a similar extent in the Emph and Emph+ MP hamsters. In a previous study, using the same dose of MP in normal rats, MP also decreased diaphragm force production without a decrease in type IIx fibre CSA while type I and IIa fibres were mildly atrophied [8]. This decrease in force was in part explained by the reduction in the number of type IIb fibres. A 3-week treatment with 6 $\mathrm{mg} \cdot \mathrm{kg}^{-1}$ prednisolone resulted in type IIx and IIb fibre atrophy, a reduction in type IIb isoforms and a decrease in force production in rat diaphragm compared to controls [27]. Other investigators, using ATPase-based fibre typing showed no effect of prednisolone [28] and MP [29] on normal rat diaphragm fibre size and composition, whereas type I, IIa and IIb fibre atrophy was observed following administration of cortisone acetate $\left(10 \mathrm{mg} \cdot \mathrm{kg}^{-1} \cdot \mathrm{day}^{-1}\right.$ for 3 weeks) in rabbits [30]. Since changes in muscle fibre morphometry cannot explain the difference in force production between the Emph and Emph+MP groups, more subtle ultrastructural changes are likely to be responsible. Ultrastructural changes such as cross-bridge kinetics, myosin heavy chain density, and energy substrates play an important role in the force generation of the muscle fibres.

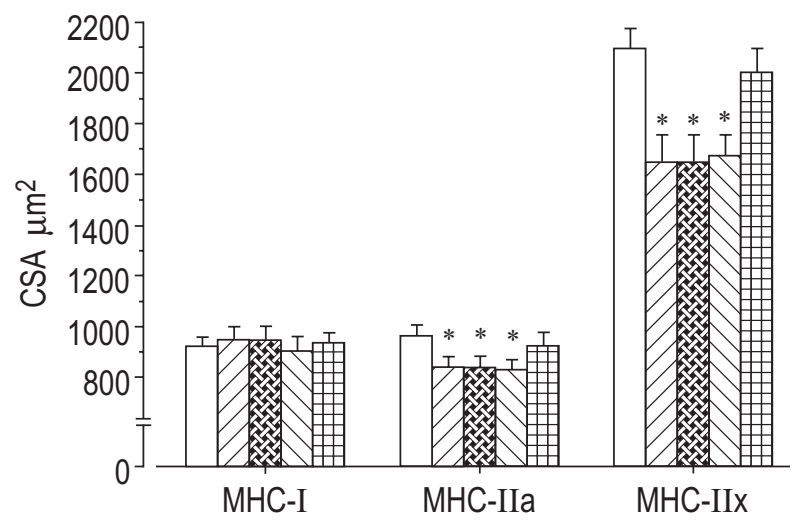

Fig. 4. - Fibre type cross-sectional area (CSA). $\square$ : control; $\mathbb{Z}$ : MP;

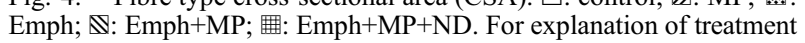
groups see figure 1. Data are shown as mean \pm SEM. $*$ : $\mathrm{p}<0.05$ compared to control and Emph+MP+ND.
Effects of nandrolone decanoate on methylprednisolonetreated emphysematous diaphragm

Several pharmacological interventions to prevent glucocorticoid-induced myopathic changes in skeletal muscles have been studied. Growth hormone treatment improved maximum inspiratory pressure and nitrogen balance in COPD patients [31]. This, however, was a preliminary report on a nonplacebo-controlled trial. In rat diaphragm, glucocorticoid-induced changes were not prevented by growth hormone administration [32]. Clenbuterol (4 mg. $\mathrm{kg}^{-1}$ over a 21 day period) [33] and testosterone (20 mg. $\mathrm{kg}^{-1} \cdot$ day $^{-1}$ ) [34] partly prevented glucocorticoid-induced muscle atrophy. In these studies, treatment with the anabolic drugs was started simultaneously with the glucocorticoid administration in order to evaluate the antagonistic potency of the anabolic drugs. ReID et al. [35] reported an increase in lean body mass and a decrease in body fat mass following testosterone replacement in prednisolone-treated $\left(\sim 10 \mathrm{mg} \cdot \mathrm{day}^{-1}\right)$ hypogonadal males. In the present study, however, the interest was whether MP-induced changes could be reversed. Since changes in diaphragm structure and function occurred following 6 months of MP treatment $\left(0.2 \mathrm{mg} \cdot \mathrm{kg}^{-1} \cdot \mathrm{day}^{-1}\right)$ [8], the hamsters in this study were treated with MP for 6 months before ND was added to the glucocorticoid treatment. ND was chosen because of the specific antagonistic action at the receptor level between synthetic anabolic steroids and glucocorticoids [36, 37]. Apart from this antagonistic mechanism, anabolic steroids have also proven to be beneficial in clinical practice [7].

Theoretically, the blunting capacity of ND on MPinduced changes may be due to a direct anabolic effect of ND on muscle fibres [3, 38, 39], or to an antagonistic action between ND and glucocorticoids. Addition of ND to Emph+MP hamsters restored $P$ t to normal control values but increased $P \max$ only to the level of the Emph hamsters. At physiological stimulation frequencies $(\sim 25$ $\mathrm{Hz}$ ), force generating capacity was similar in the Emph+ $\mathrm{MP}+\mathrm{ND}$, Emph and control groups. The increase in normalized force in the EMPH+MP+ND hamsters at 25 $\mathrm{Hz}$ can in part be the result of the increase in the sum of contraction and relaxation time of the muscle strips.

The reversibility of the loss in diaphragm force production in the Emph+MP hamsters by the addition of 
ND could be due to an anabolic effect on the emphysematous changes, to an antagonistic effect on the MPinduced changes, or a combination of both. In normal rats, however, ND completely abolished the MP-induced impairment in diaphragm force-generating capacity [9]. It is therefore most likely that ND predominantly antagonized the action of MP with at most a small effect on the changes induced by emphysema. Several mechanisms might be responsible for the interaction between anabolic steroids and glucocorticoids. Anabolic steroids are believed to act via muscle glucocorticoid receptors rather than via muscle androgen receptors in antagonizing the catabolic effects of glucocorticoids [36]. MAYER and Rosen [40] proposed a binding competition between androgens and glucocorticoids for the same site of the receptor responsible for mediating the catabolic action of glucocorticoids. Inhibition of glucocorticoid action at the gene level [41] or downregulation of glucocorticoid receptor number [37] were also reported as anabolic effects counteracting glucocorticoid-induced muscular changes. The direct effects of anabolic steroids on normal skeletal muscles include promoting amino acid incorporation into muscle proteins, decreasing amino acid catabolism [3], and increasing myosin and myofibrillar protein fraction [39]. These actions, however, apparently had at most a minor effect on emphysema induced diaphragm function impairment.

\section{Methodological considerations}

No pair-weight or pair-fed control group was added in the present study, since emphysema and the drugs tested did not affect body weight and food intake in a pilot study. Indeed, at the end of the treatment period, no changes in body weight were found among the four groups. This confirms, previous data showing no difference in body weight growth in adult hamsters $[17,18]$. Therefore, functional and structural changes as described above cannot be explained by differences in body weight. Diaphragm fibre atrophy on the morphological slides was significant but more subtle in the MP, Emph and Emph+MP groups and therefore of no major influence on diaphragm muscle mass, which did not differ between the treatment groups. The viability of the in vitro muscle preparation is not likely to be influenced by the bath temperature of $37^{\circ} \mathrm{C}$ used in the present study. SEgAL and FAULKNER [42] showed that the critical radius for oxygen diffusion was $\sim 0.6 \mathrm{~mm}$ at $37^{\circ} \mathrm{C}$. Apart from the fact that no differences in muscle strip thickness were observed, this radius is clearly greater than the radius of $0.3 \mathrm{~mm}$ used in the present study.

\section{Conclusions}

In conclusion, the decrease in force-generating capacity in the methylprednisolone-treated emphysematous diaphragm was partly antagonized by nandrolone decanoate addition. Nandrolone decanoate only antagonized the effects of methylprednisolone but did not influence diaphragmatic changes induced by emphysema. Myosin heavy chain class IIa and IIx diaphragm muscle fibre atrophy in the emphysema plus methylprednisolone group was com- pletely reversed by nandrolone decanoate. Since both methylprenisolone and nandrolone decanoate were administered in low, clinically applied dosages, these observations may support the rationale for the development of clinical trials to investigate if anabolic steroids have similar protective effects in chronic obstructive pulmonary disease patients with steroid-induced respiratory muscle dysfunction.

Acknowledgements. The authors would like to thank M.B. Reid for his comments on a previous version of this manuscript and Y. Brom for her expert biotechnical assistance.

\section{References}

1. Gibson J. Respiratory muscles. In: Calverley PMA, Pride NB, eds. Chronic Obstructive Pulmonary Disease. London, Chapman and Hall, 1995; pp. 185-203.

2. Decramer M, de Bock V, Dom R. Functional and histologic picture of steroid-induced myopathy in chronic obstructive pulmonary disease. Am J Respir Crit Care Med 1996; 153: 1958-1964.

3. Haupt HA, Rovere GD. Anabolic steroids: a review of the literature. Am J Sports Med 1984; 12: 469-484.

4. Ruff RL. Endocrine myopathies. In: Engel AG, Banker BQ, eds. Myology. McGraw-Hill, 1986; pp. 1871-1906.

5. Hudson LD, Monti CM. Rationale and use of corticosteroids in chronic obstructive pulmonary disease. Med Clin North Am 1990; 74: 661-690.

6. Laaban J, Kouchakji B, Dore M, Orvoen-Frija E, David P, Rochemaure J. Nutritional status of patients with chronic obstructive pulmonary disease and acute respiratory failure. Chest 1993; 103: 1362-1368.

7. Schols AMWJ, Soeters PB, Mostert R, Pluymers RJ, Wouters EFM. Physiologic effects of nutritional support and anabolic steroids in patients with chronic obstructive pulmonary disease - a placebo-controlled randomized trial. Am J Respir Crit Care Med 1995; 152: 1268-1274.

8. van Balkom RHH, Dekhuijzen PNR, Folgering HTM, et al. Effects of long-term low-dose methylprednisolone on rat diaphragm function and structure. Muscle Nerve 1997; 20: 983-990.

9. van Balkom RHH, Dekhuijzen PNR, Folgering HTM, et al. Anabolic steroids in part reverse glucocorticoidinduced alterations in rat diaphragm. J Appl Physiol 1998; 84: 1492-1499.

10. Snider GL, Sherter CB. A one-year study of the evolution of elastase-induced emphysema in hamsters. J Appl Physiol 1977; 43: 721-729.

11. Lewis MI, Zhan W, Sieck GC. Adaptations of the diaphragm in emphysema. J Appl Physiol 1992; 72: 934-943.

12. Farkas GA, Roussos C. Adaptability of the hamster diaphragm to exercise and/or emphysema. J Appl Physiol 1982; 53: 1263-1272.

13. Jasani MK. Anti-inflammatory steroids: mode of action in rheumatoid arthritis and homograft reaction. In: Vane JR, Ferreira SH, ed. Anti-inflammatory Drugs. Berlin, Springer-Verlag, 1979; pp. 598-660.

14. Sarett LH, Patchett AA, Steelman SL. The effects of structural alteration on the anti-inflammatory properties of hydrocortisone. Progr Drug Res 1963; 5: 11-153.

15. Moore BJ, Miller MJ, Feldman HA, Reid MB. Diaphragm atrophy and weakness in cortisone-treated rats. $J$ Appl Physiol 1989; 67: 2420-2426.

16. Frenkel JK, Havenhill MA. The corticoid sensitivity of 
Golden hamsters, rats, and mice. Lab Invest 1963; 12 : 1204-1220.

17. Kelsen SG, Wolanski T, Supinski GS, Roessmann U. The effect of elastase-induced emphysema on diaphragmatic muscle structure in hamsters. Am Rev Respir Dis 1983; 127: 330-334.

18. Mercer RR, Crapo JD. Structural changes in elastic fibers after pancreatic elastase administration in hamsters. $J$ Appl Physiol 1992; 72: 1473-1479.

19. Prakash YS, Fournier M, Sieck GC. Effects of prenatal undernutrition on developing rat diaphragm. J Appl Physiol 1993; 75: 1044-1052.

20. Schiaffino S, Gorza L, Sartore S, et al. Three myosin heavy chain isoforms in type 2 skeletal muscle fibers. $J$ Musc Res Cell Motility 1989; 10: 197-205.

21. Lewis MI, Monn SA, Zhan WZ, Sieck GC. Interactive effects of emphysema and malnutrition on diaphragm structure and function. J Appl Physiol 1994; 77: 947-955.

22. Younes M. Load responses, dyspnea, and respiratory failure. Chest 1990; 97: 59s-68s.

23. Kandarian SC, White TP. Mechanical deficit persists during long-term muscle hypertrophy. J Appl Physiol 1990; 69: 861-867.

24. Farkas GA, Gosselin LE, Zhan WZ, Schlenker EH, Sieck GC. Histochemical and mechanical properties of diaphragm muscle in morbidly obese Zucker rats. $J$ Appl Physiol 1994; 77: 2250-2259.

25. Faulkner JA, Maxwell LC, Brook DA, Lieberman DA. Adaptation of guinea pig plantaris muscle fibers to endurance training. Am J Physiol 1971; 221: 291-297.

26. Farkas GA, Roussos C. Histochemical and biochemical correlates of ventilatory muscle fatigue in emphysematous hamsters. J Clin Invest 1984; 74: 1214-1220.

27. van Balkom RHH, Zhan WZ, Prakash YS, Dekhuijzen PNR, Sieck GC. Corticosteroid effects on isotonic contractile properties of rat diaphragm muscle. J Appl Physiol 1997; 83: 1062-1067.

28. Dekhuijzen PNR, Gayan-Ramirez G, de Bock V, Dom R, Decramer M. Triamcinolone and prednisolone affect contractile properties and histopathology of rat diaphragm differently. J Clin Invest 1993; 92: 1534-1542.

29. Dekhuijzen PNR, Gayan-Ramirez G, Bisschop A, et al. Rat diaphragm contractility and histopathology are affected differently by low dose treatment with methylprednisolone and deflazacort. Eur Respir J 1995; 8: 824-830.
30. Ferguson GT, Irvin CG, Cherniack RM. Effects of corticosteroids on respiratory muscle histopathology. Am Rev Respir Dis 1990; 142: 1047-1052.

31. Pape GS, Friedman M, Underwood LE, Clemmons DR. The effect of growth hormone on weight gain and pulmonary function in patients with chronic obstructive lung disease. Chest 1991; 99: 1495-1500.

32. Petrof BJ, Gottfried SB, Eby J, LaManca J, Levine S. Growth hormone does not prevent corticosteroid-induced changes in rat diaphragm structure and function. $J$ Appl Physiol 1995; 79: 1571-1577.

33. Agbenyega ET, Wareham AC. Effect of clenbuterol on skeletal muscle atrophy in mice induced by the glucocorticoid dexamethasone. Comp Biochem Physiol 1992; 102A: 141-145.

34. Ferguson GT. Effects of cortisone and testosterone on diaphragmatic function and biochemistry in the rabbit. $J$ Appl Physiol 1995; 78: 1459-1468.

35. Reid IR, Wattie DJ, Evans MC, Stapleton JP. Testosterone therapy in glucocorticoid-treated men. Arch Intern Med 1996; 156: 1173-1177.

36. Danhaive PA, Rousseau GG. Evidence for sex-dependent anabolic response to androgenic steroids mediated by muscle glucocorticoid receptors in the rat. $J$ Steroid Biochem 1988; 29: 575-581.

37. Sharpe PM, Buttery PJ, Haynes NB. The effects of manipulating growth in sheep by diet or anabolic agents on plasma cortisol and muscle glucocorticoid receptors. Br J Nutr 1986; 56: 289-304.

38. Griggs RC, Kingston W, Jozefowicz RF, Herr BE, Forbes $\mathrm{G}$, Halliday D. Effect of testosterone on muscle mass and muscle protein synthesis. J Appl Physiol 1989; 66: 498-503.

39. Rogozkin VA. Anabolic steroid metabolism in skeletal muscle. J Steroid Biochem 1979; 11: 923-926.

40. Mayer M, Rosen F. Interaction of glucocorticoids and androgens with skeletal muscle. Metabolism 1977; 26: 937-945.

41. Hickson RC, Czerwinski SM, Falduto MT, Young AP. Glucocorticoid antagonism by exercise and androgenicanabolic steroids. Med Sci Sports Exerc 1990; 22: 331340.

42. Segal SS, Faulkner JA. Temperature-dependent physiological stability of rat skeletal muscle in vitro. Am J Physiol 1985; 248: C265-C270. 